\title{
Evolución microestructural de composites SiC/aleaciones CuSi obtenidos a través de infiltración reactiva ${ }^{(\bullet)}$
}

\author{
J. Cornejo*, S. Ordoñez* e I. Iturriza ${ }^{(b) * *}$
}

\begin{abstract}
Resumen
Se estudió la evolución microestructural de composites de SiC/aleaciones $\mathrm{Cu}-\mathrm{Si}$ obtenidos a través del proceso de infiltración reactiva a $1400^{\circ} \mathrm{C}$. Se detectaron tres zonas en los composites obtenidos: zona de reacción, transición e infiltrada. En la zona de reacción y de transición, la microestructura resultante estuvo compuesta por una fase metálica, láminas de grafito y partículas de $\mathrm{SiC}$. Se encontró que el $\mathrm{SiC}$ se descompone en estas zonas por efecto de la aleación $\mathrm{Cu}$-Si, por lo que el silicio disponible forma una solución líquida que a temperatura ambiente estuvo formada por una solución sólida $\alpha$ y una fase $\gamma\left(\mathrm{Cu}_{5} \mathrm{Si}\right)$. El carbono resultante de la descomposición del $\mathrm{SiC}$ precipitó como láminas de grafito. Además, la descomposición del $\mathrm{SiC}$ fue disminuyendo a medida que la cantidad de silicio en la aleación inicial se incrementó.
\end{abstract}

\section{Microstructural evolution of $\mathrm{SiC} / \mathrm{Cu}$-Si composites obtained through reactive infiltration}

\begin{abstract}
The microstructural evolution of composites of $\mathrm{SiC} / \mathrm{Cu}$-Si alloys obtained through process of reactive infiltration to $1400{ }^{\circ} \mathrm{C}$ was studied. Three zones were detected in the obtained composites: the reaction zone, the transition zone and the infiltrated zone. In the reaction zone and transition zone the resulting microstructure was composed of a metallic phase, graphite laminae and $\mathrm{SiC}$ particles. It was found that $\mathrm{SiC}$ decomposes into these areas because of the alloy $\mathrm{Cu}-\mathrm{Si}$, so the available $\mathrm{Si}$ forms a liquid solution that a room temperature consisted of a $\alpha$ solid solution and a $\gamma$ phase $\left(\mathrm{Cu}_{5} \mathrm{Si}\right)$. The carbon resulting from the decomposition of $\mathrm{SiC}$ precipitated as graphite laminae. In addition, the $\mathrm{SiC}$ decomposition was decreasing as the initial amount of $\mathrm{Si}$ in the alloy increased.
\end{abstract}

Keywords

Reactive infiltration; Decomposition SiC; Composite SiC/Cu; Microestructure.

\section{INTRODUCCIÓN}

La alta resistencia al desgaste, el bajo peso específico, la alta rigidez y el bajo coeficiente de dilatación térmica hacen al $\mathrm{SiC}$ un material muy atractivo para aplicaciones en ingeniería ${ }^{[1]}$. Sin embargo, la nula tenacidad tanto al impacto como a la fractura y la baja resistencia al choque térmico se presentan como las dos principales desventajas de este material. Por el contrario, los metales de transición poseen altos valores de tenacidad y, en especial, los del grupo IB tienen excelente conductividad térmica y eléctrica.
Desde la Segunda Guerra Mundial se han desarrollado los materiales compuestos. Dentro de este grupo de materiales están los composites cerámico-metal (CCM). Los CCM son redes interconectadas en el espacio de ambas fases. Esto conduce a una estructura bien diferenciada con respecto a los composites con refuerzos en forma de partículas, fibras cerámicas reforzadas o cerámicas con inclusiones metálicas. Las técnicas más utilizadas para su fabricación son infiltración reactiva, infiltración por presión isostática en caliente (HIP) y sinterización $^{[2]}$. En ellas, el metal debe tener una buena mojabilidad sobre el substrato y, en lo posible, una

(•) Trabajo recibido el día 13 de agosto de 2009 y aceptado en su forma final el día 23 de noviembre de 2009.

* Dpto. de Ingeniería Metalúrgica, Facultad de Ingeniería, Universidad de Santiago de Chile. Av. B. O’Higgins 3363, Santiago, Chile.

** CEIT. Paseo Manuel de Larrizabal 15 (20009), San Sebastian España. 
baja reactividad, con el objetivo de evitar que el substrato (cerámico) sea degradado por efecto de las reacciones químicas entre éste y el metal.

El sistema $\mathrm{SiC} / \mathrm{Cu}$ ha sido muy estudiado dada la excelente conductividad térmica y eléctrica que poseen ambos materiales ${ }^{[3-5]}$. No obstante, el uso de estos composites es limitado debido a la degradación que sufre el $\mathrm{SiC}$, producto de la reacción química que ocurre en la interfase entre éste y el cobre, disminuyendo ostensiblemente las propiedades físico-químicas y mecánicas ${ }^{[6-9]}$.

En el proceso de infiltración reactiva $\mathrm{SiC} / \mathrm{Cu}$ es muy importante tener una buena mojabilidad (bajo ángulo de contacto entre el líquido y el sólido), lo cual no ocurre entre el cobre y el carburo de silicio ${ }^{[10]}$. Por otro lado, se han descrito grandes disminuciones del ángulo de mojado para estos sistemas con el sólo hecho de incorporar un aleante en el metal, por ejemplo, hierro en cobre ${ }^{[11]}$ y silicio en cobre ${ }^{[12]}$. Durante este proceso, la aleación líquida reacciona con el $\mathrm{SiC}$ para formar varios compuestos intermetálicos y/o soluciones sólidas.

Publicaciones anteriores han informado de un ángulo de contacto entre el cobre y $\mathrm{SiC}$ a $1.100^{\circ} \mathrm{C}$ de $140^{\circ}$ y con un enlace débil ${ }^{[8]}$, aunque es bien sabido que el ángulo de contacto tiene un régimen transitorio y, luego, alcanza el equilibrio ${ }^{[13]}$. Sin embargo, el ángulo de contacto obtenido entre aleaciones $\mathrm{Cu}$ $\mathrm{Si}$ y $\mathrm{SiC}$ disminuye, aproximadamente, a $40^{\circ}$ a $1.200^{\circ} \mathrm{C}[12]$.

El primer paso hacia la obtención de un CCM con propiedades mecánicas adecuadas es identificar y caracterizar las fases metálica y cerámica.

El sistema $\mathrm{SiC} / \mathrm{Cu}$ ha sido caracterizado en trabajos anteriores ${ }^{[6-10}$ y 12]. Sin embargo, se hace necesario conocer las características microestructurales de la interfase cerámico-metal, con lo cual se podrán controlar las propiedades mecánicas finales del composite, dado que se ha publicado que la nucleación y propagación de las grietas en composites similares a este sistema ocurre en la interfase cerámico-metal ${ }^{[14]}$.

Los objetivos de este trabajo son identificar las diferentes fases presentes en el composite $\mathrm{Cu}(\mathrm{Si})$ $\mathrm{SiC}$ y compararlas con las del diagrama de equilibrio de fases $\mathrm{Cu}-\mathrm{Si}$-C. Por otro lado, se hace necesario determinar la influencia del soluto $(\mathrm{Si})$ en la aleación inicial $\mathrm{Cu}(\mathrm{Si})$ sobre el porcentaje en peso de $\mathrm{Si}$ en la fase metálica final.

\section{PROCEDIMIENTO EXPERIMENTAL}

La fabricación del substrato cerámico se hizo a partir de polvos de $\alpha$-SiC, grado UF15 PREMIX (0,65\% B4C y $3 \% \mathrm{C}$ como aditivos) suministrado por
LONZA GROUP LTDA., con un tamaño promedio de partícula de $25 \mu \mathrm{m}$. Estos polvos se compactaron en una prensa uniaxial de matriz flotante con una carga aplicada de $300 \mathrm{MPa}$. Los compactos en verde fueron sinterizados en un horno JONES con elemento calefactor de grafito bajo una atmósfera de argón a $1.600^{\circ} \mathrm{C}$. La velocidad de calentamiento fue de $0,33{ }^{\circ} \mathrm{C} \cdot \mathrm{s}^{-1}$ hasta la temperatura de sinterización, siendo el tiempo de mantenimiento a dicha temperatura de $180 \mathrm{~min}$. La densidad final de los substratos cerámicos pre-sinterizados estuvo en torno al $65 \%$ de la densidad teórica (DT), por lo que había un $35 \%$ de porosidad para ser infiltrada.

Para infiltrar los substratos cerámicos, se utilizaron tres aleaciones base cobre con 1,2 y $3 \%$ en peso de silicio. Las aleaciones se prepararon en un crisol de alúmina, que fue introducido en un horno de resistencias eléctricas LINDBERG. La infiltración reactiva se realizó en un horno de inducción ASTRO con un sistema de alto vacío $\left(6,67 \times 10^{-3} \mathrm{~Pa}\right)$. La aleación $\mathrm{Cu}$ $\mathrm{Si}$ sólida se colocó encima del substrato de $\mathrm{SiC}$ y, luego, ambos se introdujeron en un crisol de alúmina. Las dimensiones del substrato fueron $45 \times 8 \times 8 \mathrm{~mm}$. La cantidad de aleación fue 4 veces el peso de aleación requerida teóricamente para llenar el $35 \%$ de porosidad, con el objetivo de estudiar también el metal residual. La velocidad de calentamiento fue de $0,33^{\circ} \mathrm{C} \cdot \mathrm{s}^{-1}$ hasta $900^{\circ} \mathrm{C}$. El tiempo de mantenimiento a esta temperatura fue de $30 \mathrm{~min}$ para permitir la recuperación del vacío; posteriormente, se continuó con el calentamiento a la misma velocidad hasta llegar a $1.400{ }^{\circ} \mathrm{C}$, temperatura a la que se mantuvo el sistema durante $180 \mathrm{~min}$.

Los ensayos de Calorimetría Diferencial de Barrido (DSC) y Termogravimetría (TGA) se realizaron con el objetivo de seguir el comportamiento de la reacción de descomposición de $\mathrm{SiC}$ con aleaciones $\mathrm{Cu}$-Si. El equipo utilizado fue un calorímetro SETARAM modelo SETSYS EVOLUTION 16-18 bajo una atmósfera de nitrógeno y con una velocidad de calentamiento de $0,167^{\circ} \mathrm{C} \cdot \mathrm{s}^{-1}$ A partir de polvos de cobre, silicio y $\mathrm{SiC}$ se fabricaron composites con 1,3 y $5 \%$ de silicio. Se consideró que el metal representaría un $35 \%$ en volumen del composite, por lo que se calcularon las cantidades porcentuales necesarias para generar los composites. Las muestras se calentaron en un crisol de alúmina. Se realizaron 4 ciclos de calentamiento-enfriamiento, con 30 min de mantenimiento a $1.400^{\circ} \mathrm{C}$.

La caracterización microestructural se hizo en un microscopio óptico y electrónico de barrido de emisión de campo (FEG-SEM) JEOL JSM-7000 F equipado con espectrometría de dispersión de energía de rayos X (EDS) para la caracterización química. Para la identificación de las fases presentes se usó la técnica de 
difracción de rayos $\mathrm{X}$ (X-RD) en un difractómetro modelo D5000 de SIEMENS con monocromador de grafito. La radiación utilizada fue $\mathrm{Ka}$ de $\mathrm{Cu}$ de $\lambda$ $1,5406 \AA ̊$.

El software Thermo-Calc y la base de datos TCFE3 se utilizaron para calcular diagramas de fases multicomponentes y la actividad del carbono en la aleación $\mathrm{Cu}-\mathrm{Si}$.

\section{RESULTADOS Y DISCUSIÓN}

\subsection{Microestructura y reactividad del $\mathrm{SiC}$ con aleaciones $\mathrm{Cu}-\mathrm{Si}$}

En la figura 1 se muestra la microestructura de un composite $\mathrm{SiC} / \mathrm{Cu}-1$ \% en peso $\mathrm{Si}$. Es posible observar claramente tres zonas, que pueden ser denominadas como: zona de reacción, zona intermedia y zona infiltrada. Similares resultados se obtuvieron para los composites $\mathrm{SiC} / \mathrm{Cu}-2$ y $3 \%$ en peso $\mathrm{Si}$. Se determinó que la zona de reacción midió en promedio $1.100 \mu \mathrm{m}$ y la zona de transición $25 \mu \mathrm{m}$. El resto de la muestra fue infiltrada completamente $(6.875 \mu \mathrm{m})$. Una vez fundida la aleación que se colocó encima del $\mathrm{SiC}$, por acción capilar, ésta, comenzó a infiltrar de acuerdo al sentido de la flecha indicado en la figura 1. En un inicio, se dio la reacción de descomposición del $\mathrm{SiC}$ hasta llegar a una composición de silicio en equilibrio que detuvo la reacción.

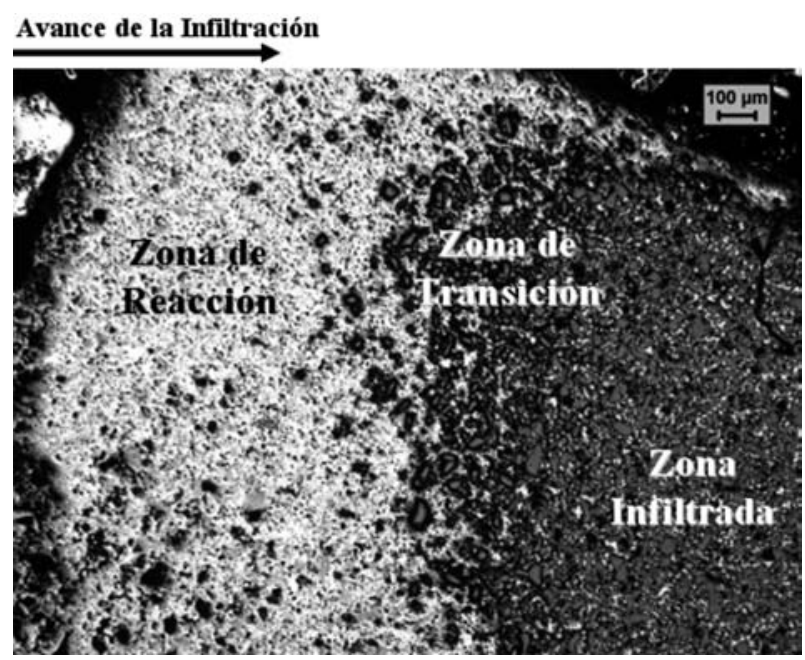

Figura 1. Micrografía óptica de un composite $\mathrm{SiC} / \mathrm{Cu}-1 \%$ en peso $\mathrm{Si}$.

Figure 1. Optical micrograph of a composite SiC/Cu-1wt \% Si.
La figura 2 muestra las micrografías obtenidas a través de FEG-SEM del composite mencionado anteriormente. Se pudo apreciar, con análisis EDS y X-RD, que la zona de reacción (Fig. 2 a)), está compuesta por una fase metálica (aleación $\mathrm{Cu}-\mathrm{Si}$ ), láminas de grafito y, en menor cantidad, partículas de $\mathrm{SiC}$. En la figura $2 \mathrm{~b}$ ) se observa una zona intermedia compuesta por una fase metálica, láminas de grafito y partículas de $\mathrm{SiC}$. A diferencia de la zona de reacción, la descomposición del $\mathrm{SiC}$ ha evolucionado de forma menos agresiva. Por último, la figura 2 c) muestra la zona infiltrada del composite, la cual está constituida de una fase metálica y partículas de $\mathrm{SiC}$ (ausencia de láminas de grafito). A simple vista, no se observa que el $\mathrm{SiC}$ esté interconectado a pesar de haber sido sinterizado. Esto se debe a que la micrografía no está hecha en tres dimensiones sino en dos.

Las microestructuras generadas en las zonas de reacción e intermedia son atribuidas a la descomposición del $\mathrm{SiC}$ en presencia de cobre, fenómeno que ya ha sido estudiado ${ }^{[6-8]}$. Al producirse la descomposición del $\mathrm{SiC}$, el silicio disponible puede generar una solución con el cobre y/o formar compuestos intermetálicos $\mathrm{Cu}-\mathrm{Si}$, dependiendo de la cantidad de silicio disponible de la reacción. Por otro lado, el carbono precipita como grafito, dada su baja solubilidad en el sistema $\mathrm{Cu}-\mathrm{Si}^{[8]}$.

\subsection{Efecto de la composición de la aleación $\mathrm{Cu}-\mathrm{Si}$ sobre la reactividad del SiC}

La figura 3 muestra el diagrama de equilibrio $\mathrm{Cu}-\mathrm{Si}-\mathrm{C}$ a $1.400{ }^{\circ} \mathrm{C}$. Se observa que la máxima cantidad de carbono disuelta en una aleación líquida $\mathrm{Cu}-\mathrm{Si}-\mathrm{C}$ a esta temperatura es $7 \mathrm{ppm}$. Sin embargo, a temperaturas inferiores a $1.100^{\circ} \mathrm{C}$, la solubilidad del carbono disminuye por debajo de $1 \mathrm{ppm}$.

En la figura 4 se muestra la evolución de la actividad del carbono (respecto al grafito) con la temperatura para diferentes aleaciones $\mathrm{Cu}$-Si iniciales. Puede apreciarse que en la medida que aumenta la cantidad de silicio en la aleación la actividad del carbono disminuye, lo que conlleva a inhibir la aparición de grafito en el sistema, puesto que la presencia de grafito se ve favorecida cuando la actividad de éste es uno (sustancia pura); valores inferiores a la unidad están asociados principalmente al SiC. También, se observa que, a temperaturas inferiores a $400^{\circ} \mathrm{C}$, la actividad del carbono en la aleación $\mathrm{Cu}$-Si es prácticamente nula, razón por la cual es posible despreciar el efecto del carbono en la fase metálica a temperatura ambiente, la que puede simplificarse y caracterizarse de acuerdo al diagrama de equilibrio binario $\mathrm{Cu}-\mathrm{Si}^{[15]}$ mostrado en la figura 5 . 

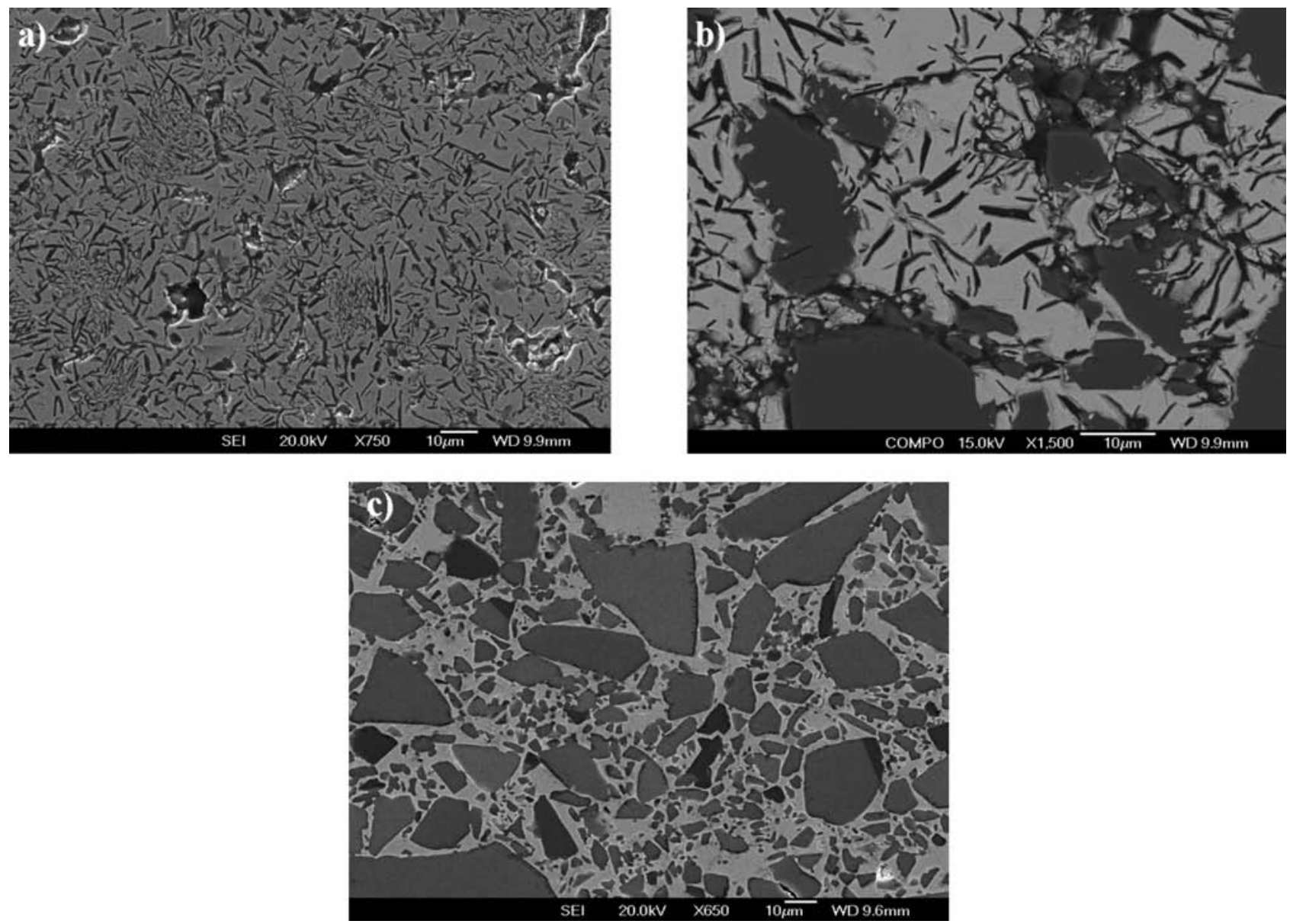

Figura 2. Micrografías FEG-SEM de un composite SiC-Cu-1\% en peso Si: (a) zona de reacción, (b) zona intermedia y (c) zona infiltrada.

Figure 2. FEG-SEM micrographs of a composite SiC/Cu-1wt \% Si: (a) reaction zone, (b) transition zone and (c) infiltrated zone.

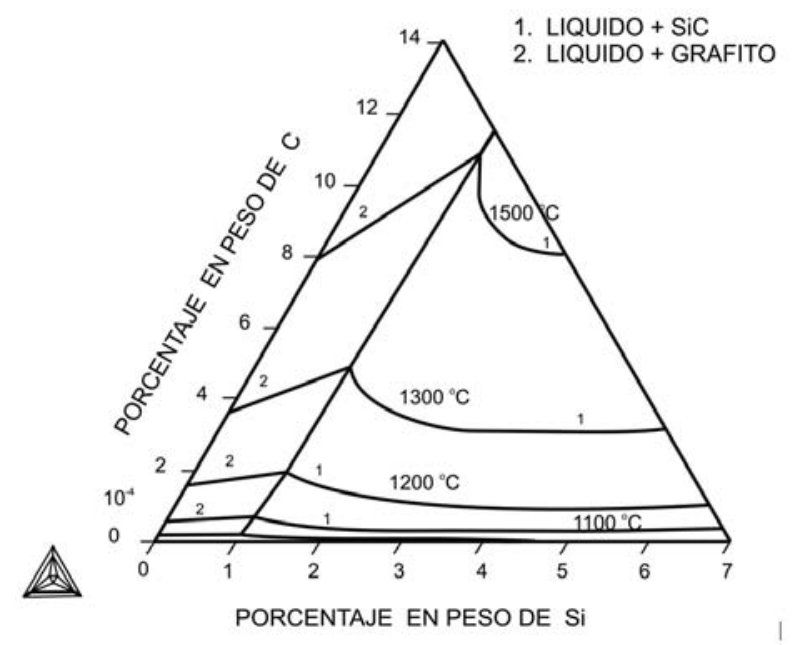

Figura 3. Diagrama de equilibrio del sistema Cu-Si-C calculado para diferentes isotermas.

Figure 3. Equilibrium diagram of the system $\mathrm{Cu}$ Si-C calculated for different isotherms.

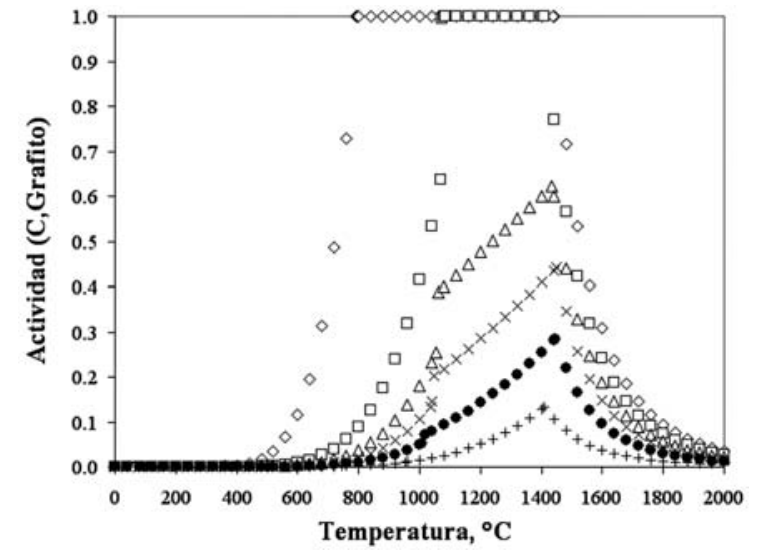

Figura 4. Evolución de la actividad del carbono (grafito) con la temperatura en algunas aleaciones Cu-Si-C.

Figure 4. Evolution of the activity of carbon (graphite) with the temperature in some alloys Cu-Si-C. 


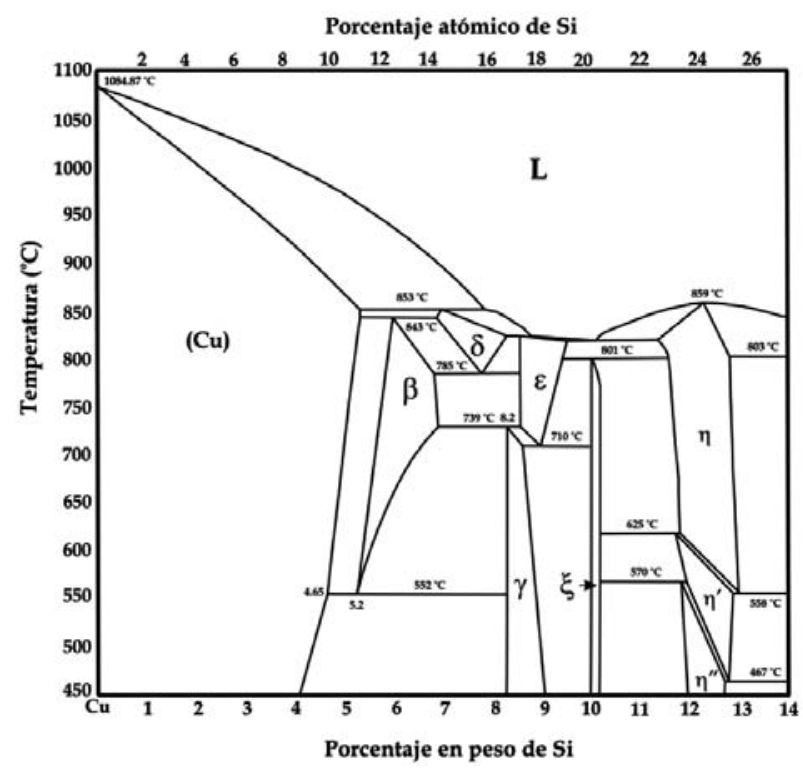

Figura 5. Diagrama de equilibrio binario de aleaciones Cu-Si[15].

\section{Figure 5. Equilibrium diagram of binary alloys $\mathrm{Cu}-\mathrm{Si}$.}

La tabla I muestra los análisis (promedios) de espectrometría de dispersión de energía (EDS) para el silicio en la matriz metálica del composite. No se observaron diferencias considerables en la cantidad de silicio en las tres zonas del composite. Por consiguiente, considerando que la matriz metálica puede caracterizarse a través del sistema $\mathrm{Cu}$-Si y utilizando la base de datos TCFE3 del software THERMOCALC para extender las líneas de interés del diagrama de equilibrio de este sistema a temperatura ambiente, es por lo que se pudieron calcular las cantidades de

Tabla I. Cantidad de Si y fase en el metal del composite

\section{Table I. Amount of silicon and phase in the metal of the composite}

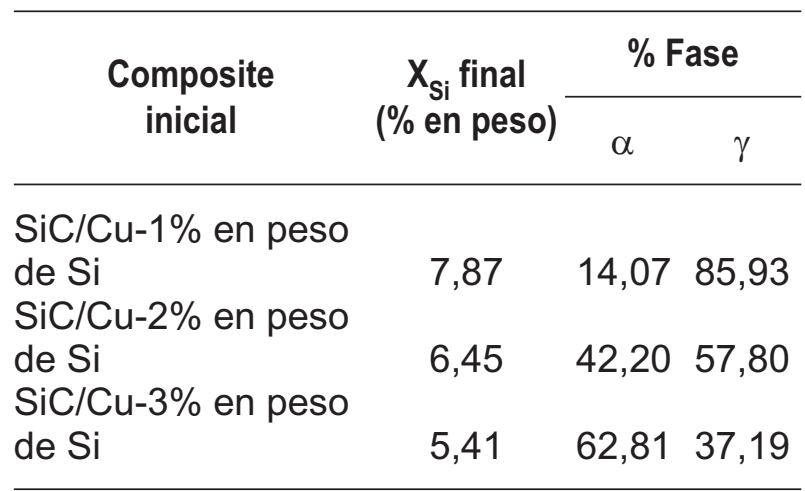

fases presentes a esta temperatura aplicando la regla de la palanca.

Se aprecia que al aumentar la cantidad de silicio en la aleación infiltrante (inicial), la cantidad de silicio en la matriz metálica del composite (final) disminuye. Esta tendencia explica la evolución de la microestructura de los composites mostrados en la figura 2, donde también se observa que a medida que se produce la descomposición del $\mathrm{SiC}$ (zona de reacción e intermedia, figuras 2 a) y b)) la aleación se va enriqueciendo en silicio, hasta llegar a un nivel en el cual se detiene esta reacción (zona infiltrada, figura 2 c)). Esto sugiere que al incrementar la cantidad de silicio en la aleación infiltrante la descomposición del $\mathrm{SiC}$ es inhibida. Por el contrario, menores cantidades de silicio generan que una mayor porción de $\mathrm{SiC}$ reaccione debido a la presencia de cobre en el sistema, por lo que la cantidad de silicio en la aleación final aumentará, como se pone de manifiesto en la tabla I.

El comportamiento anterior puede estar asociado al aumento considerable de la actividad del carbono en presencia de cobre, lo cual hace que el carbono en el $\mathrm{SiC}$ se desestabilice y llegue a su forma estable como grafito. Cuando hay cobre puro en contacto con $\mathrm{SiC}$, la actividad del carbono llega a la unidad (con respecto a una solución Raoultiana), apareciendo, de esta forma, el grafito. Al adicionar silicio al cobre (aleación) se producirá un proceso de absorción preferencial de átomos de silicio en la interfase líquido-SiC, puesto que se favorecen más los enlaces $\mathrm{Si}-\mathrm{C}$ que los $\mathrm{Cu}-\mathrm{C}^{[8]}$. Experimentalmente, se ha visto que esto provoca que el ángulo de contacto disminuya drásticamente desde $140^{\circ}$ para el Cu puro a $40^{\circ}$ para las aleaciones $\mathrm{Cu}-\mathrm{Si}^{[12]}$.

De acuerdo al diagrama de equilibrio $\mathrm{Cu}-\mathrm{Si}$ (Fig. 5), las fases presentes en el metal del composite $\mathrm{SiC}-\mathrm{Cu} / \mathrm{Si}$ serán fase $\alpha$ (solución sólida $\mathrm{Cu}-\mathrm{Si}$ ) y fase $\gamma\left(\mathrm{Cu}_{5} \mathrm{Si}\right)$. En la figura 6 se puede apreciar la evolución de los máximos de difracción para los composites $\mathrm{SiC} / \mathrm{Cu}-1$ y $3 \%$ en peso de Si. Para ambas composiciones se observa la presencia de fase $\alpha$ y $\gamma$ en la fase metálica de los composites. Esto confirma la existencia de estas fases y esta en total acuerdo con el diagrama de equilibrio binario $\mathrm{Cu}-\mathrm{Si}$.

De acuerdo con la tabla I, la fase metálica del composite tiene una mayor cantidad de silicio que la aleación inicial. En la figura 7 a) se muestra un punto triple, entre $\mathrm{SiC}$, grafito y metal que se produjo en la zona de reacción. Por otro lado, en la figura 7 b) se visualiza la fase metálica del composite correspondiente a la zona infiltrada. En esta última figura, también se observa que en la fase metálica existen pequeñas partículas con un tamaño promedio de $10 \mathrm{~nm}$. De los análisis EDS y de difracción de rayos $\mathrm{X}$ se induce que 
EVOLUCIÓN MICROESTRUCTURAL DE COMPOSITES SIC/ALEACIONES CUSI OBTENIDOS A TRAVÉS DE INFILTRACIÓN REACTIVA MICROSTRUCTURAL EVOLUTION OF SIC/CU-SI COMPOSITES OBTAINED THROUGH REACTIVE INFILTRATION

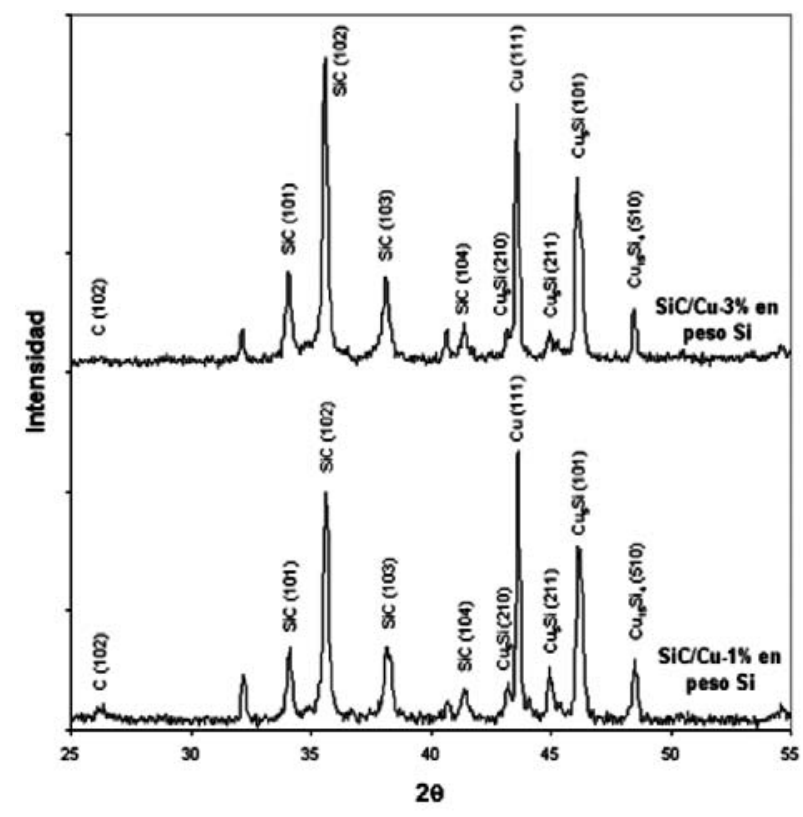

Figura 6. Difractograma correspondiente a los composites $\mathrm{SiC} / \mathrm{Cu}-1 \%$ en peso Si y $3 \%$ en peso Si.

\section{Figure 6. Diffraction patterns for the composites $\mathrm{SiC} / \mathrm{Cu}-1 \%$ by weight Si and $3 \mathrm{wt} \% \mathrm{Si}$.}

estas partículas corresponden a la fase $\gamma$ y están inmersas en una matriz cúbica de caras centradas identificada como fase $\alpha$. Por lo tanto, estos resultados coinciden con los mostrados en el difractograma de la figura 6 y la caracterización microestructural de la fase metálica de los composites predicha por el diagrama de equilibrio binario Cu-Si (Fig. 5).

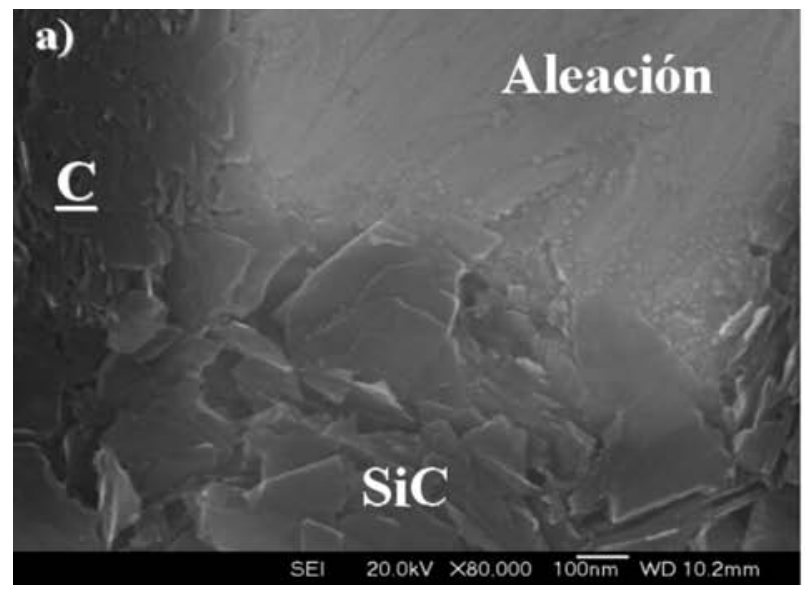

La figura 8 muestra la microestructura generada en la zona de reacción del composite $\mathrm{SiC} / \mathrm{Cu}-1$ \% en peso Si. Análisis EDS mostraron la presencia de láminas de grafito, $\mathrm{SiC}$, y una fase metálica $\mathrm{Cu}-\mathrm{Si}$ (Figs. 8 a) y 8 b)). Se aprecia que las láminas de grafito se producen a partir de la partícula de $\mathrm{SiC}$, lo cual denota un crecimiento cooperativo entre la descomposición del $\mathrm{SiC}$ y la aparición de grafito. Este efecto ha sido, asimismo, observado por otros investigadores [ ${ }^{16}$ y 17$]$, con diferentes sistemas metal/cerámico. Estos, han determinado que el crecimiento de la nueva fase ocurre en el punto triple, mientras el metal líquido está en contacto con el grafito y el $\mathrm{SiC}$ (Fig. 8 b). Además, se observa una textura en las láminas de grafito que puede estar asociada a un crecimiento epitaxial entre estas y el SiC (Fig. 8 c)). El $\alpha-\mathrm{SiC}$ utilizado tiene una estructura hexagonal $6 \mathrm{H}$ cuyo plano superficial es la familia $\{0001\}$ y el grafito presente en estos composites también exhibe una estructura hexagonal compacta.

\subsection{Análisis DSC}

Con el objetivo de profundizar en la interacción entre las aleaciones $\mathrm{Cu}-\mathrm{Si}$ y el $\mathrm{SiC}$ se ha llevado a cabo un seguimiento continuo de las reacciones que tienen lugar tanto en el calentamiento como en el enfriamiento de estos composites. Convencionalmente, se adoptó que los máximos representan transformaciones exotérmicas y los mínimos representan a las transformaciones endotérmicas. La figura 9 muestra los resultados de los análisis DSC y TGA del primer calentamiento realizados a las mezclas de polvos

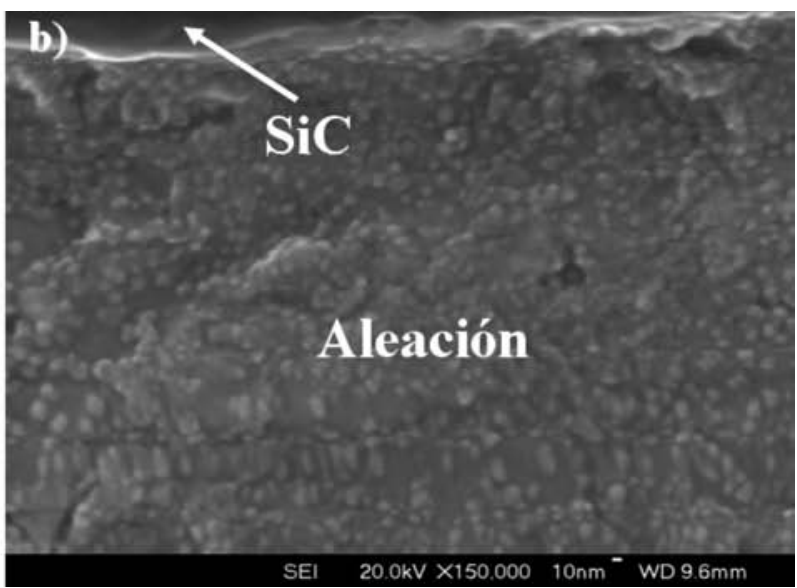

Figura 7. Micrografías FEG-SEM: (a) interfase grafito-SiC-metal de la zona de reacción y (b) fase metálica de la zona infiltrada.

Figure 7. FEG-SEM micrographs: (a) interface graphite-SiC-metal of the reaction zone and (b) metallic phase infiltrated zone. 

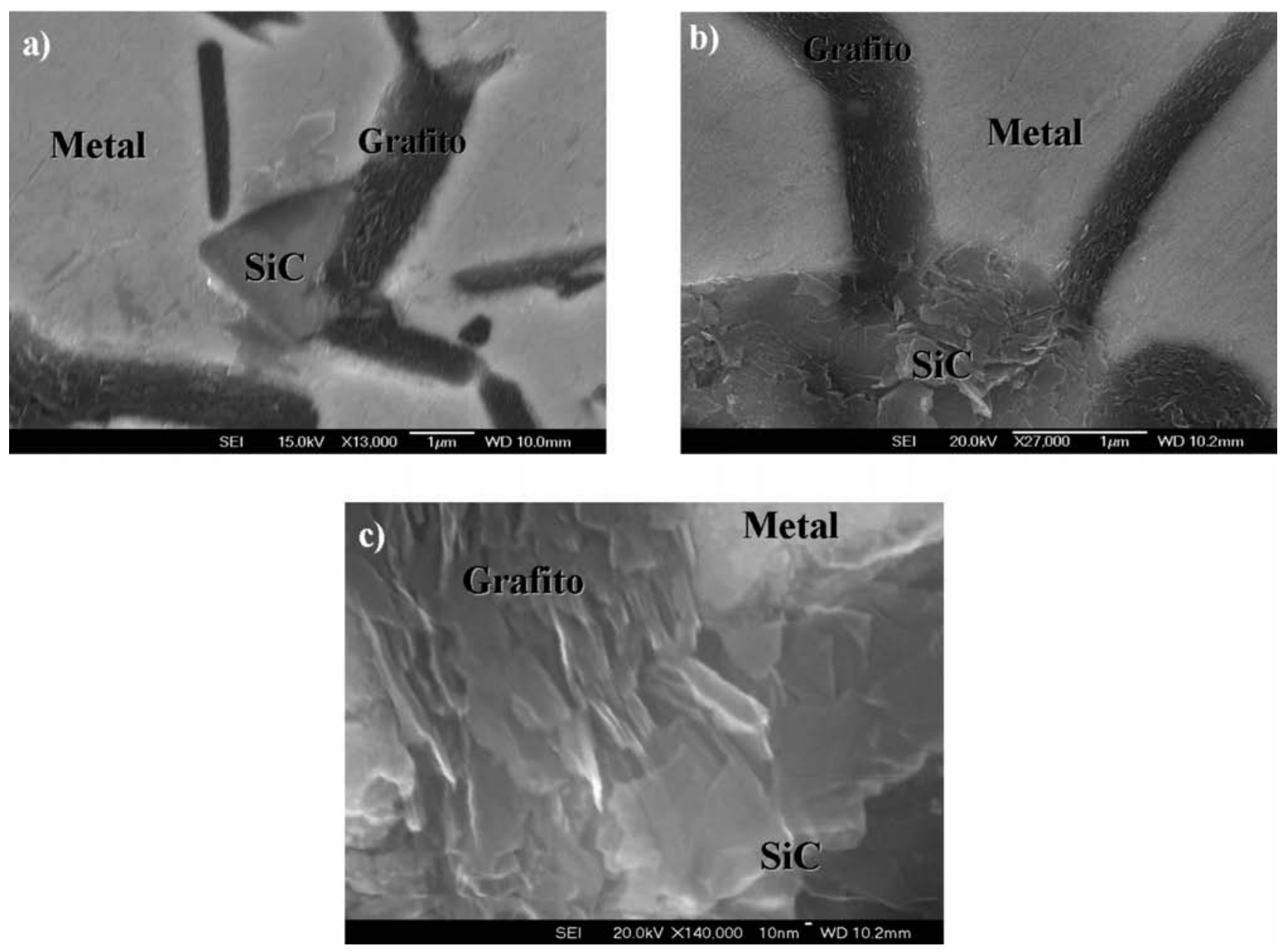

Figura 8. Micrografías FEG-SEM de la zona de reacción del composite $\mathrm{SiC} / \mathrm{Cu}-1 \%$ en peso de Si; (a) 13.000X, (b) 27.000X y (c) 140.000X.

Figure 8. FEG-SEM micrographs of the reaction zone of the composite SiC/Cu-1\% by weight Si; (a) 13,000X, (b) 27,000X and (c) 140,000X.

elementales cobre, silicio y $\mathrm{SiC}$ que representarían los composites $\mathrm{SiC} / \mathrm{Cu}-\mathrm{X} \%$ en peso de $\mathrm{Si}(\mathrm{X}=1,2$ y 5). La figura 9 a) muestra el análisis DSC de estos composites. Las fluctuaciones registradas entre temperatura ambiente y $260^{\circ} \mathrm{C}$ corresponden a la variabilidad de la fuente de poder del equipo por alcanzar lo más rápido posible una velocidad de calentamiento constante $\left(0,33{ }^{\circ} \mathrm{C} \cdot \mathrm{s}^{-1}\right)$. Entre 260 y $460^{\circ} \mathrm{C}$ se libera el aglutinante que viene incorporado en los polvos de $\mathrm{SiC}$. Estos efectos se aprecian en la grafica TGA de la figura $9 \mathrm{~b}$ ) como una fuerte pérdida de masa $(\approx 5 \%)$ durante el calentamiento entre temperatura ambiente y $460{ }^{\circ} \mathrm{C}$.

Para las mezclas con 1,2 y $5 \%$ en peso de silicio inicial se observa un máximo endotérmico entre 800 y $865^{\circ} \mathrm{C}$, aunque en la mezcla con $1 \%$ en peso de silicio es poco perceptible. Al aumentar la temperatura, se producirá una difusión superficial y volumétrica de silicio y cobre. Se sabe que la difusión superficial es mucho más rápida que la volumétri$\mathrm{ca}^{[18]}$. Esto provocará que existan zonas ricas en silicio. Las cantidades de estas zonas dependerán del porcentaje de silicio inicial en la mezcla, lo cual explica que el máximo endotérmico para la mezcla con $1 \%$ en peso de silicio sea casi imperceptible, caso opuesto al que ocurre en las mezclas con 2 y $5 \%$ en peso de silicio (Fig. 9 a)). Lo anterior está en total acuerdo con lo establecido en el diagrama de equilibrio Cu-Si (Fig. 5). Del rango de temperatura en que ocurre esta transformación y la ayuda del diagrama de equilibrio $\mathrm{Cu}-\mathrm{Si}$ se puede estimar que la fracción de líquido presente tendría en promedio un 7,7 \% en peso de silicio.

Un segundo máximo se observa en torno a $1.060-1.080^{\circ} \mathrm{C}$, como consecuencia de la fusión de las partículas de $\mathrm{Cu}\left(1.083^{\circ} \mathrm{C}\right)$. Sin embargo, la mezcla con $5 \%$ en peso de silicio inicial presenta un máximo endotérmico más débil que las otras mezclas. 

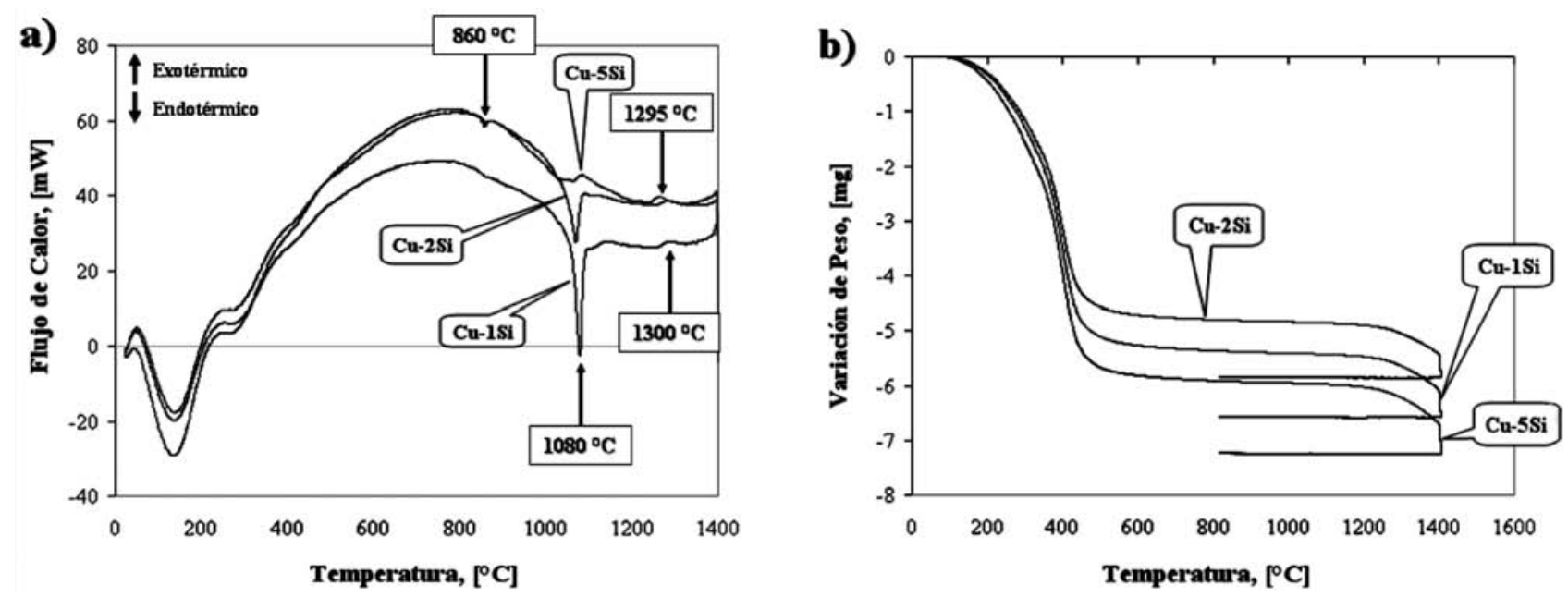

Figura 9. Análisis para el primer calentamiento de los composites $\mathrm{SiC} / \mathrm{Cu}-\mathrm{X} \%$ en peso de $\mathrm{Si}(\mathrm{X}=1,2$ y 5): (a) DSC y (b) TGA.

Figure 9. Analysis for the first heating of composites SiC / Cu-X wt \% Si (X=1, 2 and 5): (a) DSC and (b) $T G A$.

Esta aleación en estado homogéneo tiene un punto de fusión entorno a los $970{ }^{\circ} \mathrm{C}$. No obstante, la aparición del primer líquido de esta aleación ocurre alrededor de los $860^{\circ} \mathrm{C}$, por lo que este rango de temperaturas representa una zona bifásica sólido líquido. Sin embargo, se logra fundir completamente la aleación metálica a $1.077^{\circ} \mathrm{C}$ debido a la mayor presencia de partículas de cobre.

Posterior a la fusión de la fase metálica, en la figura 9 a), se observan unos pseudo máximos exotérmicos

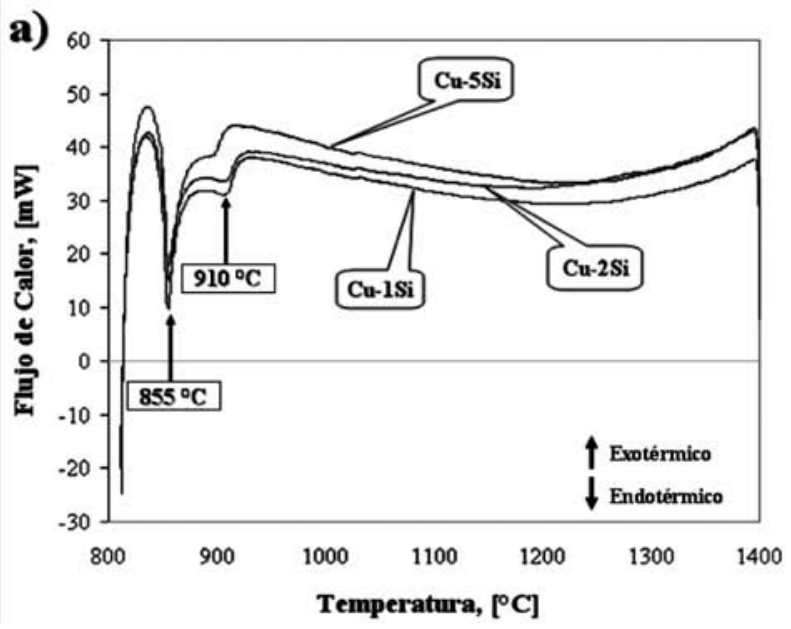

(en torno a $1.300^{\circ} \mathrm{C}$ ) los cuales pueden estar asociados a la reacción de descomposición del SiC. En los siguientes ciclos de calentamiento (segundo a cuarto) de los composites no se apreciaron estos máximos exotérmicos. La figura 10 a) muestra solo el cuarto ciclo de calentamiento, puesto que el segundo y tercero tuvieron un comportamiento similar. Lo anterior sugiere que la reacción de descomposición del SiC procede en forma masiva en el primer calentamiento, puesto que se estaría alcanzando la composición de

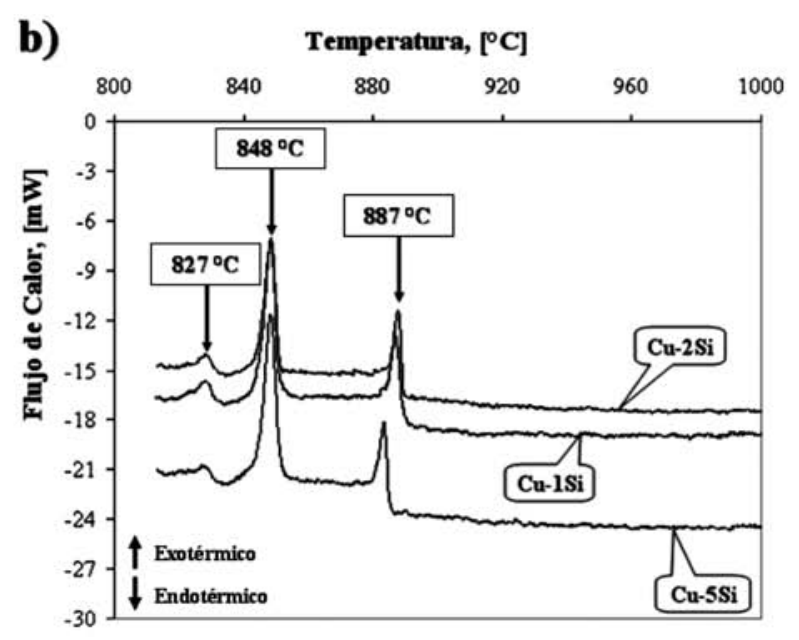

Figura 10. Análisis DSC composites $\mathrm{SiC} / \mathrm{Cu}-\mathrm{X} \%$ en peso de $\mathrm{Si}(\mathrm{X}=1 ; 2$ y 5$)$ : (a) segundo al cuarto calentamiento y (b) Cuarto enfriamiento.

Figure 10. DSC analysis of composites SiC / Cu-X wt\% Si (X=1; 2 and 5): (a) second to fourth heating and (b) fourth cooling. 
silicio en equilibrio, deteniéndola completamente $^{[7 \text { y } 8]}$. Si esto no ocurriera, se tendría que haber generado un desplazamiento de los mínimos endotérmicos ya que la aleación estaría aumentando su contenido de silicio.

Los mínimos endotérmicos $\left(852^{\circ} \mathrm{C}\right)$ de la figura 10 a) pueden atribuirse a la fusión del peritéctico $\delta(\delta \rightarrow \mathrm{L}+\alpha)^{[12]}$. El segundo mínimo endotérmico $\left(900^{\circ} \mathrm{C}\right)$ corresponde a la fusión total de la aleación $\mathrm{Cu}-\mathrm{Si}$. Por otro lado, en los ciclos de enfriamiento se observaron algunos máximos exotérmicos. El primero aparece a $885^{\circ} \mathrm{C}$, que corresponde a la solidificación de la fase $\alpha$. El segundo, puede ser atribuido a la reacción peritéctica (fase $\beta$ ) a $848^{\circ} \mathrm{C}$. El último máximo exotérmico está asociado a la precipitación de la fase $\beta$, que ocurre a $827^{\circ} \mathrm{C}$. Las diferencias apreciadas entre las temperaturas de aparición de las fases en el calentamiento y enfriamiento se atribuyen a los fenómenos de solidificación de las aleaciones (subenfriamiento, nucleación, etc. ${ }^{[19]}$. Con base en el diagrama de equilibrio $\mathrm{Cu}$-Si, se estima que las composiciones de la fase metálica están entre 5,5 a $8 \%$ en peso de silicio, lo que coincide, de manera excelente, con las observaciones realizadas en FEG-SEM, análisis EDS y X-DR.

\section{CONCLUSIONES}

Se logró relacionar las fases presentes en los composites fabricados con el diagrama de equilibrio $\mathrm{Cu}-\mathrm{Si}$.

Se determinó que los composites obtenidos presentaron tres zonas características: zona de reacción, zona intermedia y una zona infiltrada. La microestructura de las zonas de reacción e intermedia consistió en una matriz metálica (fase $\alpha$ y $\gamma$ ), láminas de grafito y partículas de $\mathrm{SiC}$. Para la zona infiltrada, la microestructura estuvo formada por partículas de $\mathrm{SiC}$ y una matriz metálica compuesta con las mismas fases $(\alpha$ y $\gamma)$.

La descomposición de $\mathrm{SiC}$ con aleaciones base cobre tiende a disminuir con el incremento del contenido de silicio en la aleación inicial.

\section{Agradecimientos}

Los autores expresan su reconocimiento a la Dirección de Investigaciones Científicas y Tecnológicas (DICYT) de la Universidad de Santiago de
Chile y a FONDECYT (Proyecto № 1030059) por el apoyo financiero otorgado para la realización de este trabajo. Además, se agradece al proyecto MECESUP UCH0205, por su invaluable apoyo económico tanto en becas de mantenimiento como en las pasantías de investigación realizadas.

\section{REFERENCIAS}

[1] C. D. Qin y B. Derby, British. Cer. Trans. J. 90 (1991) 124-125.

[2] A. M. Davidson y D. Regener, Compos. Sci. Technol. 60 (2000) 865-869.

[3] A. Brendel, C. Popescu y C. Leyens, J. Nucl. Mater. 329-333 (2004) 804-808.

[4] K. M. Shu y G. C. Tu, Mat. Sci. Eng. A 349 (2003) 236-247.

[5] G. Sundberg, P. Paul, C. Sung y T. Vacilos, J. Mater. Sci. 41 (2003) 236-247.

[6] S. Ordoñez, V. Martínez, F. Castro, L. Olivares y J. Marín, J. Mater. Sci. 38 (2003) 4.047-4.054.

[7] J. Cornejo, Tésis Magister, Facultad de Ingeniería, Universidad de Santiago de Chile, 2005.

[8] C. Rado, B. Drevet y N. Eustathopoulos, Acta Mater. 48 (2000) 4.483-4.491.

[9] H. Sakao y J. Elliot, Metall. Trans. 5 (1974) 2.036.

[10] C. Rado, S. Kalogeropoulou y N. Eustathopoulos, Mat. Sci. Eng. A 276 (2000)195-202.

[11] K. Gan, M.Y. Gu y G. Mu, J. Mater. Sci. 43 (2008) 1.318-1.323.

[12] C. Rado y N. Eustathopoulos, Interface Sci. 12 (2004) 85-92.

[13] K. Landry, C. Rado y N. Eustathopoulos, Metall. Mater.Trans. 27A (1996) 3.181-3.186.

[14] M. Song y B. Huang, Mat. Sci. Eng A 488 (2008) 601-607.

[15] Smithells y Colin J., Metal Reference Book, Vol. II, Ed. Elseiver Inc., 4⿳亠丷厂 Ed., Oxford, Inglaterra, 1967, pp. 11-264.

[16] Y. Naidich, Prog. Surf. Membrane Sci. 14 (1981) 353.

[17] I. Aksay, C. Hoge, y J. Pask, J.Phys. Chem. 78 (1974) 1.178.

[18] R. German, Powder Metallurgy Science, $2^{\text {nd }} E d$., College Road East., Princeton, New Jersey, EE. UU., 1994, pp. 15-120.

[19] J.D. Verhoeven, Fundamentals of Physical Metallurgy, Ed. John Wiley\&Sons, Nueva York, EE. UU., 1975, pp. 592. 